\title{
Bayesian heterogeneous assembly time modeling for robotic performance prediction
}

\author{
Mingyang LI*, Xuxue SUN*, Guoyuan LIANG**, Yingjun SHEN***, Qingpeng ZHANG ${ }^{\dagger}$ \\ and Yachun $\mathrm{FENG}^{* *}$ \\ * Department of Industrial and Management Systems Engineering, University of South Florida \\ Tampa, FL 33612, USA \\ ** Center for Intelligent and Biomimetic Systems, Shezhen Institutes of Advanced Technology, Chinese Academy of Sciences \\ Shenzhen, 518055, China \\ E-mail: gy.liang@siat.ac.cn

Received: 10 October 2019; Revised: 28 September 2020; Accepted: 12 January 2021

\begin{abstract}
Robotic systems are widely applied in manufacturing industry to reduce labor cost and increase productivity. Due to the influence of different observed factors (e.g., controllable robotic settings) and the unobserved heterogeneity caused by the unobserved/unknown factors (e.g., material non-uniformity, geometry variability of assembly units) during the assembly process, the assembly time of assembly units exhibits highly heterogeneous performance. To improve the productivity of the assembly process, it is important to develop an analytics-based model to provide accurate assembly time prediction of assembly units and further identify the potential influencing factors for further process improvement. This paper proposes a full Bayesian modeling approach to predicting the performance of heterogeneous robotic assembly time data. Specifically, a generic statistical model is provided to characterize the heterogeneous assembly time data of a heterogeneous population of assembly units by considering the influence of both observed factors as well as unobserved heterogeneity. Bayesian sampling algorithm is further developed to address a series of parameters estimation challenges, such as highly correlation among parameters from different sub-populations and non-conjugate priors. With the developed Bayesian estimation algorithm, both the influence of observed factors as well unobserved heterogeneity can be jointly quantified with both point estimates and exact internal estimates obtained. Both the numerical and case studies are further provided to justify the validity of the proposed modeling approach and demonstrate its superior prediction performance. The proposed prediction model with considering the population heterogeneity of assembly units exhibits better assembly time prediction performance as compared to alternative modeling approaches with the restrictive assumption of population homogeneity. Moreover, the proposed model is able to identify the relevant factors and quantify their influence with parameters uncertainty quantification for further performance improvement of the assembly process.
\end{abstract}

Keywords : Bayesian modeling, Population heterogeneity, Hazard regression, Gibbs sampler, Process improvement

\section{Introduction}

Over the past years, tremendous advancements in automation technology have enabled a new generation of manufacturing system (RoboticsVO,2013). Various kinds of robots have been designed for performing dirty, dangerous, repetitive as well as monotonous work, and widely used across the domains of manufacturing industry, space mission, health care and military defense (Prez et al., 2020). Particularly in the field of manufacturing, robots with high precision and reliability have almost replaced the human mechanics in many factories (Barbosa et al., 2020). This not only occurs in large 
manufacturing enterprises but also in small or medium sized companies trying to cope with the fierce market competition and satisfy the highly dynamic market demands of miniaturized, complex and customized products with short life cycles (Molina et al., 2005; Li et al., 2014b).

As a key technology to manufacturing industries, performance evaluation of the robotic assembly system is critical to reliable and efficient production and provides the basis for subsequent system performance improvement (Kimble et al., 2020; Weng et al., 2019). In this paper, we focus on how to predict the performance of the robotic assembly process. Defining the key performance indices is a crucial step in performance measurement. Hinckeldeyn et al., describes and organizes a set of assembly performance measurements in reference to a taxonomy of assembly skills and tasks (Hinckeldeyn et al., 2010; Chen et al., 2013). Li et al., proposed that the assembly throughput curve is used to identify the assembly bottlenecks and respective optimization potentials ( $\mathrm{Li}$ et al., 2014a). Most of applications of robots in the manufacturing industries have been primarily implemented as fixed processes of automatic operations, in which the same sequence of actions is repeated hundreds or thousands of times (Chen et al., 2013). Cheng et al., investigated the modeling problem for robotic assembly processes using Gaussian Process Regression, a non-parametric modeling technique to model the relationship between the assembly process parameters and performance (Cheng and Chen, 2014; Hollerbach et al., 2009; Montgomery, 2007). Nabi and Tauseef analyzed a mixed model flexible manufacturing system for multiple products with inter-cellular and intra-cellular routing flexibility, and introduced a colored Petri net formalism model for modelling and evaluation of a carousel configured cellular production system with possible variation in manufacturing and assembly resources (Nabi and Tauseef, 2020). The performance of such robotic systems can be measured by different performance indices, such as quality, reliability and productivity (Jiang et al., 2020; Woodall and Montgomery, 1999). The performance indices of quality and reliability have been studied by using statistical process control and reliability engineering for several decades (Shi, 2006; Elsayed, 2000; Lee and Wang, 2003; Sumanth, 1984; Sheng et al., 2014; Yang et al., 2015; Nilakantan et al., 2015; Raj et al., 2012). Productivity is also desirable because it is closely interrelated with system quality and reliability (Li et al., 2014b). According to (Li et al., 2014b) and (Raj et al., 2012), assembly time is a variable directly related to the productivity of the assembly process, which defines how long a robot will take to accomplish an assigned assembly task. Actually, assembly time is affected by a series of factors, such as robotic control parameters, etc. (Raj et al., 2012). An accurate model that mathematically links the impacts of those factors on robotic assembly time will provide the basis for identifying significant influencing factors and determining appropriate control parameters to improve system performance. In order to improve the productivity of robotic assembly process, particle swarm optimization (Levitin et al., 2006; Marvel et al., 2009) and genetic algorithms (Xing and Wang, 2012; McLachlan and Krishnan, 2007; Diebolt and Robert, 1994) were applied to the robotic assembly process modeling and thus to minimize the robotic assembly time.

Because of various types of uncertainties in the robotic assembly process, to our knowledge, a general and adaptive method by which the impacts of different possible productivity influencing factors can be accurately quantified is still lacking for analyzing robotic assembly time. In (Li et al., 2014b), the observations of robotic assembly time are assumed to be independently and identically distributed. Since observed data often exhibits heterogeneity, the homogeneity assumption may be too idealized. By explicitly quantifying the heterogeneity of observation data, a more accurate model has been proposed in (Raj et al., 2012). The heterogeneity may be caused by quality heterogeneity of assembly units, such as parts dimensions and raw-material quality. However, in (Raj et al., 2012), their influences on the assembly time is considered. It is also possible that some of these factors may contribute to the classification of assembly units into different assembly time sub-populations.

In this paper, a novel statistical model is proposed to characterize the heterogeneous robotic assembly time. Both the observed heterogeneity caused by various observed factors and the unobserved heterogeneity caused by the unobserved or unknown factors are quantified. In particular, for the observed productivity influencing factors, their effects in influencing assembly time as well as classifying assembly time into different sub-populations are simultaneously investigated. The proposed model will help practitioners in evaluating and predicting the productivity of robotic assembly system at any time point probabilistically. In addition, the quantified effects of different possible productivity influencing factors will also facilitate calibration of robotic settings for further productivity improvement of the assembly process. A real case study of an assembly production process is provided to demonstrate the effectiveness of the proposed method.

The remainder of this paper is organized as follows: Section 2 introduces the proposed model formulation, addresses the corresponding model estimation challenges and describes the proposed estimation algorithm; Section 3 illustrates the proposed model and demonstrates its validity through both a numerical case study and a real case study. Section 4 draws the conclusions. 


\section{Methodology}

Due to many observed (e.g., controllable robotic settings) and unobserved factors' (e.g., material non-uniformity, geometry variability of assembly units) influence on assembly units during the assembly process, the assembly time of units tends to be highly heterogeneous. To account for such data heterogeneity and improve model prediction performance, a generic statistical model formulation is presented in this section to characterize the heterogeneous assembly time data of assembly units. Specifically, considering a heterogeneous population of units with $m$ homogeneous sub-populations, the probability distribution function of the assembly time $f(t)$ can be represented as

$$
f(t \mid x, y, \Theta)=\sum_{j=1}^{m} w_{j}\left(y ; \Phi_{j}\right) f_{j}\left(t \mid x, \theta_{j}\right), j=1, \cdots, m
$$

where $t$ is the assembly time of a unit from the overall heterogeneous population, $f_{j}(t)$ and $\omega_{j}$ are the probability density function and the corresponding mixing proportion for the $j^{\text {th }}$ homogeneous sub-population, $j=1, \cdots, m, \theta_{j}$ and $\Phi_{j}$ are collections of all unknown parameters in $f_{j}(t)$ and $\omega_{j}$, respectively. $x$ and $y$ are $p_{x} \times 1$ and $p_{y} \times 1$ covariate vectors that represent the possible observed factors that may affect assembly time of units from each sub-population as well as the associated sub-population proportion, respectively. $\Theta$ represents a collection of all unknown parameters in Eq. (1), i.e., $\Theta=\left\{\theta_{j}, \Phi_{j}\right\}_{j=1}^{m}$.

A popular choice of such functional mapping is the logistic function, which can be expressed as

$$
w_{j}\left(y ; \alpha_{j}\right)=\frac{\exp \left(\alpha_{j}^{T} y\right)}{1+\Sigma_{j=1}^{m-1} \exp \left(\alpha_{j}^{T} y\right)}, j=1, \ldots, m-1
$$

and $\omega_{m}=1-\Sigma_{j=1}^{m-1} \omega_{j}=1 /\left(1+\sum_{j=1}^{m-1} \exp \left(\alpha_{j}^{T} y\right)\right)$, where $\alpha_{j}$ is a $\left(p_{y}+1\right) \times 1$ covariate coefficient vector and $\Theta_{j}=\alpha_{j}$. Thus, $\omega_{j}$ 's can be modeled by the multinomial logistic regression model as

$$
\log \left(\frac{\omega_{j}}{\omega_{m}}\right)=\alpha_{j}^{T} y, j=1, \ldots, m-1
$$

To specify the model structure of $f_{j}(t)$ by explicitly considering the influence of $x$, hazard regression model can be employed to capture the hazard rate of the $j^{\text {th }}$ sub-population and is given by

$$
h_{j}(t)=h_{j}^{b}(t) \exp \left(\beta_{j}^{T} x\right), j=1, \ldots, m
$$

where $h_{j}^{b}(t)$ is the baseline hazard function which captures the inherent hazard rate of units from the $j^{t h}$ sub-population when $\beta_{j}=0$. There exists an unique relationship between $h_{j}(t)$ and $f_{j}(t)$ and the latter can thus be determined as

$$
f_{j}(t)=h_{j}(t) \exp \left(-\int_{0}^{t} h(s) d s\right), j=1, \ldots, m
$$

$h_{j}^{b}(t)$ can be specified parametrically and a widely-accepted parametric form is $h_{j}^{b}(t)=\frac{k_{j}}{\eta_{j}}\left(\frac{t}{\eta_{j}}\right)^{k_{j}-1}$, where $k_{j}$ and $\eta_{j}$ are the corresponding shape and scale parameters of the $j^{\text {th }}$ sub-population. The aforementioned Eqs. (1), (3) and (4) constitute the proposed model of modeling assembly time of a robotic assembly process. $\omega_{j}$ 's quantify the unobserved heterogeneity and $x$ and $y$ quantify the influence of possible covariates on the process performance.

Given the assembly time data, i.e., $D=\left\{t_{i}, \delta_{i}, x_{i}, y_{i}\right\}_{i=1}^{n}$, of $n$ assembly units from a heterogeneous population, the unknown parameters in the proposed model can be estimated. $t_{i}, x_{i}$ and $y_{i}$ represent the observed assembly time, assembly time influencing factors and sub-population proportion influencing factors, respectively, of unit $i^{\text {th }}$ and $\delta_{i}$ is a right-censored indicator. Ideally, if the time allowed for data collection is unlimited, the time of finishing assembling a unit can always be recorded. In real practice, however, experiment time is restricted due to time and/or cost constraints. Thus, a fixed pre-determined terminated time, denoted as $t_{c}$, is set for recording assembly time of each unit. If assembly process is finished within $t_{c}, \delta_{i}=1$ and $t_{i}$ is the exact assembly time for the $i^{\text {th }}$ unit; otherwise, $\delta_{i}=0$ and $t_{i}=t_{c}$. Based on the available data, the joint data likelihood function can be expressed as

$$
L(\Theta \mid D)=\prod_{i=1}^{n}\left(f\left(t_{i} \mid \Theta\right)\right)^{\delta_{i}}\left(1-F\left(t_{i} \mid \Theta\right)\right)^{1-\delta_{i}}
$$

where $F(t \mid \Theta)$ is the cumulative distribution function and $\Theta$ is a collection of all unknown parameters described in Eqs. (1), (3) and (4), i.e., $\Theta=\left\{w_{j}, k_{j}, \eta_{j}, \alpha_{j}, \beta_{j}\right\}_{j=1}^{m}$. It is challenging to make statistical inference directly based on Eq. (6) for both 
Bayesian and non-Bayesian approaches since $L(\theta \mid D)$ can be expanded into $m^{n}$ additive terms and it is computationally formidable to compute integrals and/or derivatives $m^{n}$ times separately even when $n$ is moderate. For the non-Bayesian approaches, estimation method such as Expectation-Maximization can be employed to avoid maximizing $L(\Theta \mid D)$ directly and the computational complexity can be significantly reduced (see (McLachlan and Krishnan, 2007) and references therein for more details). This paper focuses on the full Bayesian approach in estimating the unknown parameters. As compared to the non-Bayesian approaches, Bayesian approach possesses several advantages in both modeling and parameters estimation. For example, it formally introduces the concept of prior distribution and makes it possible to quantify and incorporate the prior knowledge into modeling. For estimation, it can accommodate the small sample size while for the non-Bayesian approach, it requires a large sample to make certain asymptotic conditions hold.

With the prior independence assumed for all unknown parameters, the joint posterior of all unknown parameters, $\pi(\Theta \mid D)$ is given by

$$
\pi(\Theta \mid D)=\prod_{i=1}^{n}\left\{\left(f\left(t_{i} \mid \Theta\right)\right)^{\delta_{i}}\left(1-F\left(t_{i} \mid \Theta\right)\right)^{1-\delta_{i}}\right\} \prod_{j=1}^{m}\left\{\pi\left(k_{j}\right) \pi\left(\eta_{j}\right) \pi\left(\alpha_{j}\right) \pi\left(\beta_{j}\right)\right\}
$$

To obtain the posteriors of unknown model parameters under the Bayesian framework, it requires multiple integrations to marginalize out the rest of unknown parameters in Eq. (7). Similar to Eq. (6), Eq. (7) can also be expressed as a sum of $m^{n}$ additive terms. Thus, such multiple integrals need to be performed on $m^{n}$ terms separately, which is computationally challenging for standard numerical integration methods. To directly implement the Markov Chain Monte Carlo (MCMC) methods, such as the Gibbs sampling, in generating the empirical samples of their marginal posteriors, the complex form of the joint posterior still makes it computationally difficult. Specifically, Gibbs sampling requires alternately generating empirical sample points for each unknown parameter from its full conditional posterior density. The complex form of Eq. (7) yields the complex form of the corresponding full conditional posterior density for each unknown parameter. Such complexity includes (i) function inverse which is difficult to compute and make sampling methods, such as inversion sampling, inapplicable; (ii) highly dependency among all unknown parameters, which makes the Gibbs sampling inefficient. For example, the full conditional posterior of $k_{j}$ can be expressed as

$$
\pi\left(k_{j} \mid \mathrm{D}, \Theta^{\left(-k_{j}\right)}\right) \propto \prod_{i=1}^{n}\left\{\left(\sum_{j=1}^{m} w_{j}\left(\alpha_{j}\right) f_{j}\left(t_{i} \mid k_{j}, \eta_{j}, \beta_{j}\right)\right)^{\delta_{i}}\left(1-\sum_{j=1}^{m} w_{j}\left(\alpha_{j}\right) F_{j}\left(t_{i} \mid k_{j}, \eta_{j}, \beta_{j}\right)\right)^{1-\delta_{i}}\right\} \pi\left(k_{j}\right)
$$

where $\Theta^{\left(-k_{j}\right)}$ is a collection of unknown parameters which $k_{j}$ does not depend on and $\Theta^{\left(-k_{j}\right)}=\left\{k_{j}, \eta_{j}, \beta_{j}\right\}_{j=1}^{m} \cup\left\{\alpha_{j}\right\}_{j=1}^{m-1} / k_{j}$. Inverse of Eq. (8) with respect to $k_{j}$ is difficult to obtain and $k_{j}$ depends on all the rest of unknown parameters. To address the aforementioned estimation difficulties, we consider the data augmentation techniques (Diebolt and Robert, 1994) in the developed Gibbs sampling algorithm. The rationale is to introduce the latent variables $z_{i}$ 's for observations $t_{i}$ 's. $z_{i}$ represents the membership of $t_{i}$ and follows categorical distribution as $f\left(z_{i}\right)=\prod_{j=1}^{m} w_{j}^{1\left(z_{i}=j\right)}$ where $1(\cdot)$ is an indicator function. Conditioned on $z_{i}$ 's, the estimation procedure can be greatly simplified and summarized as

Step 1: Initialize unknown parameters as $\Theta^{(0)}$ and latent variables as, i.e., $\left\{z_{i}^{(0)}\right\}_{i=1}^{n}$. For any iteration $\tau=1, \cdots, \tau_{m}$, repeat steps 2-5;

Step 2: Draw $z_{i}^{(\tau)}$ from $\pi\left(z_{i} \mid D, \Theta^{(\tau-1)}\right), i=1, \cdots, n$;

Step 3: Draw $k_{j}^{(\tau)}$ from $\pi\left(k_{j} \mid D, z_{i}^{(\tau)}, \eta_{j}^{(\tau-1)}, \beta_{j}^{(\tau-1)}\right), j=1, \ldots, m$;

Step 4: Draw $\eta_{j}^{(\tau)}$ from $\pi\left(\eta_{j} \mid D, z_{i}^{(\tau)}, k_{j}^{(\tau)}, \beta_{j}^{(\tau-1)}\right), j=1, \ldots, m$;

Step 5: Draw $\beta_{j}^{(\tau)}$ from $\pi\left(\beta_{j} \mid D, z_{i}^{(\tau)}, k_{i}^{(\tau)}, \eta_{i}^{(\tau)}\right), j=1, \cdots, m$;

Step 6 : Draw $\alpha_{j}^{(\tau)}$ from $\pi\left(\alpha_{j} \mid D, z_{i}^{(\tau)},\left\{\alpha_{j}^{(\tau)}\right\}_{j^{\prime}=1}^{j-1},\left\{\alpha_{j}^{(\tau)}\right\}_{j^{\prime}=j+1}^{m-1}\right), j=1, \cdots, m-1$;

In step $2, \pi\left(z_{i} \mid D, \Theta^{(\tau-1)}\right)=\prod_{j=1}^{m}\left(q_{i, j}^{(\tau-1)}\right)^{1\left(z_{i}=j\right)}$, where $q_{i, j}^{(\tau-1)}$ is given by

$$
q_{i, j}^{(\tau-1)}=\frac{f_{j}\left(t_{i} \mid k_{j}^{(\tau-1)}, \eta_{j}^{(\tau-1)}, \beta_{j}^{(\tau-1)}\right)^{\delta_{i}}\left(1-F_{j}\left(t_{i} \mid k_{j}^{(\tau-1)}, \eta_{j}^{(\tau-1)}, \beta_{j}^{(\tau-1)}\right)\right)^{1-\delta_{i}}}{f\left(t_{i} \mid \Theta^{(\tau-1)}\right)^{\delta_{i}}\left(1-F\left(t_{i} \mid \Theta^{(\tau-1)}\right)\right)^{1-\delta_{i}}} \cdot w_{j}\left(y_{i}, \alpha_{j}^{(\tau-1)}\right)^{(\tau-1)}
$$

Since $z_{i}$ has the density of categorical distribution, $z_{i}^{(\tau)}$ can be generated conveniently. In steps 3-6, the full conditional densities, in general, do not belong to any common distributions, and specific sampling techniques, such as MetropolisHasting sampling, is required to generate the corresponding samples. It is noticed that in step 3, with the help of latent variables $z_{i}$ 's, the number of dependent parameters for $k_{j}$ are significantly reduced as compared to Eq. (8). In summary, by exploiting the structure of Eq. (7) and introducing the data augmentation techniques in the developed Gibbs sampling 
algorithm, unknown model parameters within each sub-population are decoupled and become conditionally independent, which facilitate the Bayesian sampling of these parameters. Further, by incorporating the more general purpose sampling algorithm of Metropolis-Hasting algorithm into the developed Gibbs sampling framework (e.g., Steps 3-6), the model parameters with non-conjugate priors can be more conveniently sampled as well.

\section{Case studies}

\subsection{Numerical case study}

To illustrate the proposed model and demonstrate its validity, a numerical case study is first provided based on the synthetic data generated from a heterogeneous population. Without losing generality, assuming that the heterogeneous population consists of two homogeneous sub-populations. Further, the synthetic data of each sub-population is generated from a Weibull regression with a single covariate $x$ and the mixing proportion is linked with a logistic regression with a single covariate $y$. Table 1 summaries the pre-determined model parameters. They will serve as ground truth values and evaluate the accuracy of the estimated parameters learned from the developed Bayesian estimation algorithm in Section 2. For simplicity, covariate coefficients are set as the same for two sub-populations, i.e. $\beta_{1}=\beta_{2}=\beta$. Based on such settings, a random sample of 500 observations is generated.

Table 1 Simulation settings.

\begin{tabular}{l|l|l|l|l|l|l|l|l|l}
\hline$\alpha_{0}$ & $\alpha_{1}$ & $k_{1}$ & $k_{2}$ & $\eta_{1}$ & $\eta_{2}$ & $\beta$ & $t_{c}$ & $x$ & $y$ \\
\hline-30 & 2.5 & 0.7 & 3 & $2 . \mathrm{E}+03$ & $8 . \mathrm{E}+04$ & 1 & $1 . \mathrm{E}+05$ & $\mathrm{U}(0,2)$ & $\mathrm{U}(0,15)$ \\
\hline
\end{tabular}

Based on such data, Bayesian inference is adopted to estimate the proposed model as described in Section 2. Uniform priors are assumed for unknown parameters and Table 2 shows the estimation results. Both results of point estimates (i.e., posterior mean and posterior median) and interval estimates (i.e., credible intervals) are satisfactory, which indicate the effectiveness of the developed Bayesian estimation algorithm in correctly estimating the model parameters. Further, 95\% credible intervals (CIs) fully cover the ground truth values assumed in Table 1.

Table 2 Estimation results.

\begin{tabular}{l|l|l|l|l}
\hline Para. & True Value & Posterior Mean & Posterior Median & $95 \%$ CI \\
\hline$\alpha_{0}$ & -30 & -31.4 & -31.07 & {$[-42.03,-22.97]$} \\
$\alpha_{1}$ & 2.5 & 2.64 & 2.61 & {$[1.93,3.54]$} \\
$k_{1}$ & 0.7 & 0.78 & 0.78 & {$[0.66,0.90]$} \\
$k_{2}$ & 3 & 2.9 & 2.9 & {$[2.68,3.13]$} \\
$\eta_{1}$ & $2 . \mathrm{E}+03$ & $2.19 \mathrm{E}+03$ & $2.14 \mathrm{E}+03$ & {$[1.55 \mathrm{E}+03,3.13 \mathrm{E}+03]$} \\
$\eta_{2}$ & $8 . \mathrm{E}+04$ & $7.81 \mathrm{E}+04$ & $7.81 \mathrm{E}+04$ & {$[7.31 \mathrm{E}+04,8.34 \mathrm{E}+04]$} \\
$\beta$ & 1 & 0.96 & 0.96 & {$[0.79,1.11]$} \\
\hline
\end{tabular}

To make further comparison between the proposed model with the correct model structure and other mis-specified models, two alternative model structures are assumed, denoted as "Model 1" and "Model 2". Model 1 assumes the Weibull regression with a single covariate of $x$ and neglects $y$. Model 2 also assumes the Weibull regression but with two covariates of $x$ and $y$. Both Model 1 and Model 2 assume the underlying population is homogeneous rather than heterogeneous. To compare the three models and investigate how covariates $x$ and $y$ influence the success rate curve, four different scenarios of covariate settings are considered (and shown in Figs. 1 and 2), namely $x=1, y=5, x=3, y=5, x=1, y=12$ and $x=3, y=12$. Under each scenario, the test data of 100 observations is generated and compared with predictive samples of three different models obtained from the previous training data of 500 observations. Successability curve, defined as the probability of successfully assembling an assembly unit till time $t$, i.e. $P_{r}(T \leq t)$, is displayed to compare the performance of three models and illustrate the influence of $x$ and $y$ on the successability of the assembly process. Successability curve of test data is utilized as the benchmark for model comparison. As shown in both Figs. 1 and 2 , the prediction results of the proposed model are much closer to test data curves as compared to those prediction results based on alternative models (e.g., Model 1 and Model 2). The prediction underperformances of Model 1 and Model 2 are essentially due to their simplified assumption of population homogeneity. They assume all data observations captured by the same underlying homogeneous population parameters. Such models are much restrictive as compared to the more generic model formulation of the proposed work in characterizing population heterogeneity, which allows data observations captured by different underlying homogeneous sub-population parameters. 


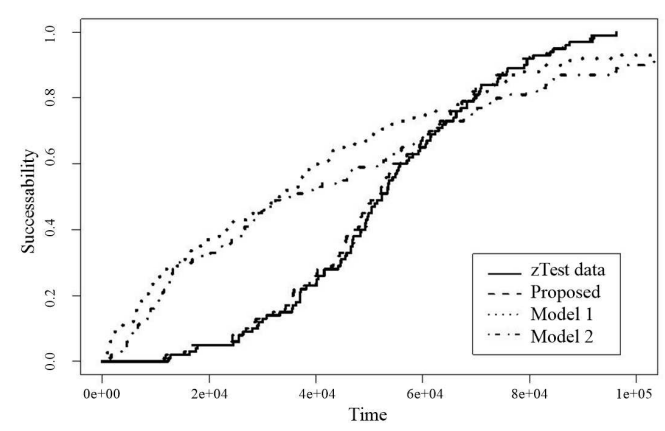

(a) Comparison at $x=1, y=5$

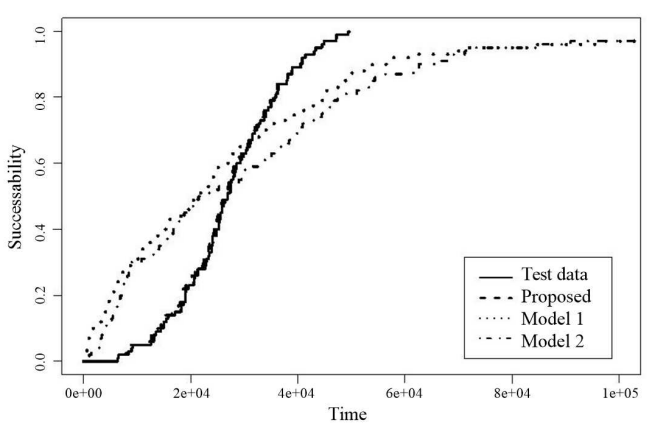

(b) Comparison at $x=3, y=5$

Fig. 1 Prediction comparison based on test data of scenarios 1 and 2

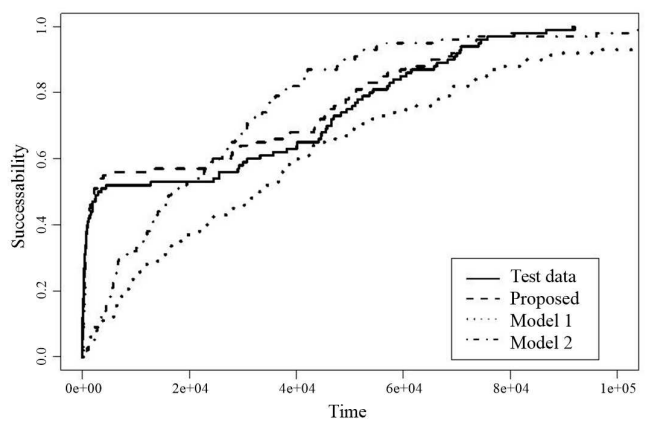

(a) Comparison at $x=1, y=12$

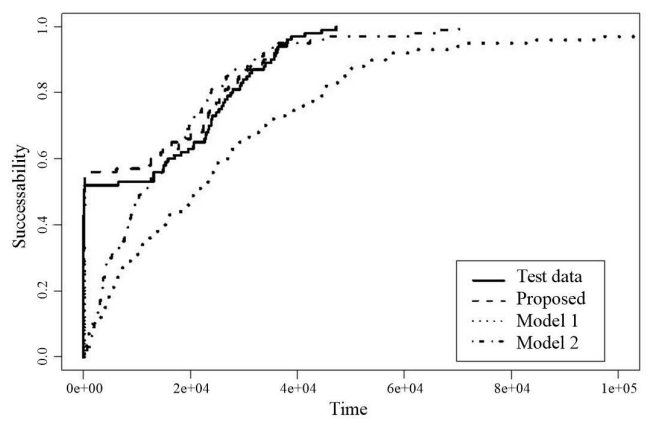

(b) Comparison at $x=3, y=12$

Fig. 2 Prediction comparison based on test data of scenarios 3 and 4

\subsection{Real case study}

A real case study of a robotic assembly system is further presented to demonstrate the importance and effectiveness of the proposed model applied in real practice. The robotic assembly system consists of an ABB IRB140 robot with an IRC5 controllers ( $\mathrm{Li}$ et al., 2014a). The assembly task is to assemble a population of valve units to the value body. Since the assembly parts are manufactured from different batches with different clearance and geometry variations, the assembly time is highly heterogeneous. Further, different robotic settings (e.g., force) may also affect the assembly time. The proposed model with two sub-populations is considered to characterize the heterogeneous assembly time data by taking into account the influence of both the batch differences as well as robotic settings.

Two variables, namely "V1" and "V2" are selected for model construction based on the domain knowledge of expertise. V1 is a controllable variable representing the force parameter of robot during the valve assembly process. V2 represents different batches. Due to the confidentiality issue, the detailed information and value of each variable is masked.

Three models are considered by treating V1 as $x$ and V2 as $y$. The proposed model structure is closer to K-M curve as compared to the alternative models, as shown in Fig. 3. It is noticed that the probability quantity along the vertical axis refers to the probability of successfully assembling a valve unit.

\section{Conclusion}

In this paper, we propose a full Bayesian model to characterize and quantify both the observed heterogeneity caused by various observed factors and the unobserved heterogeneity caused by the unobserved or unknown factors on robotic assembly time. The influence of observed factors on both assembly time as well as mixing proportions of sub-populations are simultaneously quantified. Unobserved heterogeneity is also quantified by assuming the sub-populations membership of each assembly time is unobserved. Prior knowledge of domain expertise is also possible to be incorporated into the 


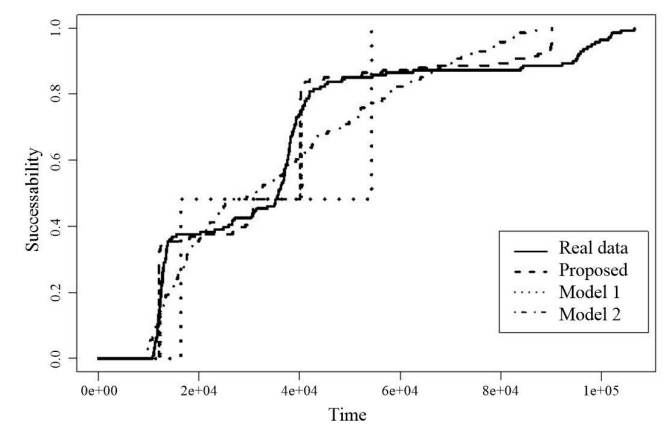

Fig. 3 Comparison based on real data

Bayesian model formulation. Gibbs sampling with data augmentation method is employed to resolve the computational difficulty. To illustrate the proposed model and demonstrate its validity, both numerical case study and real case study are proposed to illustrate our method and demonstrate its performance. By identifying and quantifying the observed heterogeneity and unobserved heterogeneity, the proposed work will help practitioners to develop better calibration plans and productivity growth strategies to improve the assembly process performance.

\section{Acknowledgement}

This work is partially supported by Joint Funds of the National Natural Science Foundation of China with Shenzhen City (No.U1813209, No. U1913211) and Science and Technology Service Network Initiatives of Chinese Academy of Sciences (KFJ-STS-QYZX-095).

The authors would like to thank Prof. Jian Liu's comments on the algorithm described in this paper as well as Dr. Heping Chen for providing data for experiments.

\section{References}

Barbosa, W. S., Gioia, M. M., Natividade, V. G., Wanderley, R. F., Chaves, M. R., Gouvea, F. C. and Gonalves, F. M., Industry 4.0: examples of the use of the robotic arm for digital manufacturing processes. International Journal on Interactive Design and Manufacturing (IJIDeM) (2020), pp.1-7.

Chen, H., Cheng, H., Liu, J., Zhang, B., Zhang, G. and Fuhlbrigge. T., Performance improvement for high accuracy assembly process in manufacturing automation. Proceedings of IEEE International Conference on Automation Science and Engineering (CASE)(2013), pp.540-545.

Cheng, H. and Chen, H., Online parameter optimization in robotic force controled assembly processes. Proceedings of IEEE International Conference on Robotics and Automation (ICRA)(2014), pp.3465-3470.

Diebolt, J. and Robert, C. P., Estimation of finite mixture distributions through bayesian sampling. Journal of the Royal Statistical Society. Series B(Methodological) (1994), pp.363-375.

Elsayed, E. A., Invited paper perspectives and challenges for research in quality and reliability engineering. International Journal of Production Research, Vol.38,No.9(2000), pp.1953-1976.

Hinckeldeyn, J., Kubera, D., Altfeld, N. and Kreutzfeldt, J., Measuring the performance of assembly processes using throughput curves, Proceeding of the 19th International Conference on Management of Technology. International Association for Management of Technology (IAMOT)(2010).

Hollerbach, J., Mason, M. and Christensen, H., A roadmap for us robotics: from internet to robotics,Tech. rep., Computing Community Consortium (CCC) (2009).

Jiang, J., Huang, Z., Bi, Z., Ma, X. and Yu, G., State-of-the-Art control strategies for robotic PiH assembly. Robotics and Computer-Integrated Manufacturing, Vol.65 (2020), 101894.

Kimble, K., Van Wyk, K., Falco, J., Messina, E., Sun, Y., Shibata, M., ... and Yokokohji, Y., Benchmarking Protocols for Evaluating Small Parts Robotic Assembly Systems. IEEE Robotics and Automation Letters, Vol.5, No.2 (2020), pp.883-889.

Lee, E. T. and Wang, J., Statistical methods for survival data analysis(2003), John Wiley \& Sons. 
Levitin, G., Rubinovitz, J. and Shnits. B., A genetic algorithm for robotic assembly line balancing. European Journal of Operational Research, Vol.168, No.3 (2006), pp.811-825.

Li, B., Cheng, H., Chen, H. and Jin, T., Modeling complex robotic assembly process using Gaussian process regression. Proceedings of 9th IEEE Conference on Industrial Electronics and Applications(2014a), pp.456-461.

Li, M., Chen, H., Zhang, B., Liu, J. and Kim, B. U., Hazard regression modeling for robotic performance prediction, Proceedings of the 2014 Industrial and Systems Engineering Research Conference, Institute of Industrial Engineers(2014b), pp.3465-3471.

Marvel, J. A., Newman, W. S., Gravel, D. P., Zhang, G., Wang, J. and Fuhlbrigge, T., Automated learning for parameter optimization of robotic assembly tasks utilizing genetic algorithms. Proceedings of IEEE International Conference on Robotics and Biomimetics (ROBIO)(2009), pp.179-184.

McLachlan, G. and Krishnan, T., The EM algorithm and extensions(2007), Vol.382, John Wiley \& Sons.

Molina, A., Rodriguez, C. A., Ahuet, H., Corts, J. A., Ramrez, M., Jimnez, G. and Martinez, S., Next generation manufacturing systems: key research issues in developing and integrating reconfigurable and intelligent machines. International Journal of Computer Integrated Manufacturing (IJCIM), Vol.18,No.7 (2005), pp.525-536.

Montgomery., D. C., Introduction to statistical quality control(2007), John Wiley \& Sons.

Nabi, Hafiz Zahid, and Tauseef Aized., Performance evaluation of a carousel configured multiple products flexible manufacturing system using Petrinet. Operations Management Research (2020), pp.1-21.

Nilakantan, J. M., Huang, G. Q. and Ponnambalam, S. G., An investigation on minimizing cycle time and total energy consumption in robotic assembly line systems. Journal of Cleaner Production, Vol.90 (2015), pp.311-325.

Prez, L., Rodrguez-Jimnez, S., Rodrguez, N., Usamentiaga, R., Garca, D. F. and Wang, L., Symbiotic humanrobot collaborative approach for increased productivity and enhanced safety in the aerospace manufacturing industry. The International Journal of Advanced Manufacturing Technology, Vol.106, No.3-4 (2020), pp.851-863.

Raj, M. V., Sankar, S. S. and Ponnambalam, S. G., Particle swarm optimization algorithm to maximize assembly efficiency. The International Journal of Advanced Manufacturing Technology, Vol.59, No.5-8 (2012), pp.719-736.

RoboticsVO. A roadmap for us robotics: From internet to robotics:2013 edition. Accessed on line, available from [http://www.robotics-vo.us/node/332], (accessed in 2013).

Sheng, Z., Shi, W. and Xiu, C., Reliability analysis method study of multistate system based on fuzzy Bayesian network. Open Automation and Control Systems Journal, Vol.6 (2014), pp.601-608.

Shi, J., Stream of variation modeling and analysis for multistage manufacturing processes(2006). CRC press.

Sumanth, D. J., Productivity engineering and management: productivity measurement, evaluation, planning, and improvement in manufacturing and service organizations(1984). McGraw-Hill College.

Weng, C. Y., Tan, W. C., Yuan, Q. and Chen, I. M., Quantitative assessment at task-level for performance of robotic configurations and task plans. Journal of Intelligent \& Robotic Systems, Vol.96, No.3-4 (2019), pp.439-456.

Woodall, W. H. and Montgomery, D. C., Research issues and ideas in statistical process control. Journal of Quality Technology, Vol.31, No.4 (1999), pp.376.

Xing, Y. and Wang, Y., Assembly sequence planning based on a hybrid particle swarm optimization and genetic algorithm. International Journal of Production Research, Vol.50, No.24 (2012), pp.7303-7312.

Yang, H., Li, M., Han, J., Chen, H., Zhang, B. and Liu, J., Statistical modeling of heterogeneous robotic assembly time with weibull regression. In: Proceedings of IEEE International Conference on Robotics and Biomimetics (ROBIO)(2015), pp.710-714. 